\title{
Radio recombination lines from the starburst galaxy NGC 3256
}

\author{
A. L. Roy ${ }^{1,2,3,4,5}$, W. M. Goss ${ }^{4}$, Niruj R. Mohan ${ }^{6}$, and K. R. Anantharamaiah ${ }^{5, \star}$ \\ 1 Max-Planck-Institut für Radioastronomie, Auf dem Hügel 69, 53121 Bonn, Germany \\ e-mail: aroy@mpifr-bonn.mpg.de \\ 2 Geodätisches Institut der Universität Bonn, Nussallee 17, 53115 Bonn, Germany \\ 3 Australia Telescope National Facility, PO Box 76, Epping 1710, NSW, Australia \\ ${ }^{4}$ NRAO, PO Box O, Socorro, NM 87801, USA \\ 5 Raman Research Institute, CV Raman Ave, Sadashivanagar, Bangalore 560080, India \\ ${ }^{6}$ Institut d'Astrophysique de Paris, 98bis boulevard Arago, 75014 Paris, France
}

Received 10 August 2004 / Accepted 14 February 2005

\begin{abstract}
We have detected the radio recombination lines $\mathrm{H} 91 \alpha$ and $\mathrm{H} 92 \alpha$ with rest frequencies of $8.6 \mathrm{GHz}$ and $8.3 \mathrm{GHz}$ from the starburst nucleus NGC 3256 at an angular resolution of 16.4" $\times 9.6^{\prime \prime}$ using the Australia Telescope Compact Array and at an angular resolution of $12.0^{\prime \prime} \times 2.9^{\prime \prime}$ using the VLA. The line was detected at $\sim 1 \mathrm{mJy}^{-1}$ beam $^{-1}$ peak with a width of $160 \mathrm{~km} \mathrm{~s}^{-1}$ with the ATCA and at $\sim 0.5 \mathrm{mJy} \mathrm{beam}^{-1}$ peak with a width of $114 \mathrm{~km} \mathrm{~s}^{-1}$ with the VLA. Modelling the line emitting region as a collection of $\mathrm{H}$ II regions, we derive constraints on the required number of $\mathrm{H}$ II regions, their temperature, density, and distribution. We find that a collection of 10 to $300 \mathrm{H}$ II regions with temperatures of $5000 \mathrm{~K}$, densities of $1000 \mathrm{~cm}^{-3}$ to $5000 \mathrm{~cm}^{-3}$ and diameters of $15 \mathrm{pc}$ produced good matches to the line and continuum emmission. The Lyman continuum production rate required to maintain the ionization is $2 \times 10^{52} \mathrm{~s}^{-1}$ to $6 \times 10^{53} \mathrm{~s}^{-1}$, which requires 600 to $17000 \mathrm{O5}$ stars to be produced in the starburst.
\end{abstract}

Key words. galaxies: individual: NGC 3256 - galaxies: nuclei - radio lines: galaxies

\section{Introduction}

Starburst activity is one of the more spectacular events of galaxy evolution. Star formation is usually slow and well regulated but occasionally runs away converting much of the interstellar medium (ISM) in the host galaxy into stars in a shortlived phase. Many presently normal galaxies may once have gone through a starburst phase, which would enrich and maybe redistribute the ISM. Understanding starbursts is therefore important for understanding how galaxies came to be as they are at present.

Radio recombination lines (RRLs) offer a useful tool for probing compact nuclear starbursts since radio wavelengths pass unattenuated through the obscuring dust that hampers optical and near-infrared studies of starbursts. RRL observations also provide dynamical information with unprecedented resolution, because the lines occur at conveniently high frequencies where interferometers provide arcsec resolution.

The potential for detecting and exploiting extragalactic RRLs was shown by Shaver (1978) and shortly thereafter RRLs were detected from the starbursts in M 82 and NGC 253 in the late 1970's (Shaver et al. 1977; Seaquist \& Bell 1977). These two have since been studied over a wide range of frequencies, yielding several important constraints on the physical state and

\footnotetext{
* Deceased.
}

kinematics in the nuclear regions (e.g. Anantharamaiah \& Goss 1997; Rodriguez-Rico et al. 2004, in preparation).

Following those first two detections came a period of surveys that produced no further detections (Churchwell \& Shaver 1979; Bell \& Seaquist 1978; Bell et al. 1984). A renewed effort during the early 1990s using the VLA with improved sensitivity detected RRLs near $8.6 \mathrm{GHz}$ from several bright starburst galaxies at a level an order of magnitude weaker than the first two detections. These new detections are NGC 660, NGC 1365, NGC 2146, NGC 3628, NGC 3690, NGC 5253, M 83, IC 694, Arp 220, Henize 2-10 (Anantharamaiah et al. 1993; Zhao et al. 1996; Phookun et al. 1998; Mohan et al. 2001), NGC 1808 (Mohan 2002), and NGC 4945 at mm wavelengths (Viallefond, private communication).

During a recent RRL survey using the Australia Telescope Compact Array (ATCA), we have made three new detections: NGC 3256, NGC 4945 and the Circinus galaxy. Here, we report the detection of NGC 3256, and the other two will be presented in later papers.

NGC 3256 is a pair of colliding disk galaxies that are partly merged and display spectacular tidal tails and disrupted morphologies (e.g. Schweizer 1986). At an inferred distance of $37 \mathrm{Mpc}\left(v_{\mathrm{r}}=(2781 \pm 24) \mathrm{km} \mathrm{s}^{-1}\right.$ from optical emission lines; de Vaucouleurs et al. 1991), the FIR luminosity is $1.9 \times 10^{11} L_{\odot}$ following the method of Helou et al. (1985), making it one of 
the most luminous galaxies with a recession velocity less than $3000 \mathrm{~km} \mathrm{~s}^{-1}$. The molecular gas mass, inferred from CO emission, is extremely large $\left(3 \times 10^{10} M_{\odot}\right.$; Sargent et al. 1989). The far-infrared (FIR) colours (Rowan-Robinson \& Crawford 1989), and the near-infrared colours (Glass \& Moorwood 1985) are typical of starburst galaxies. The $\mathrm{Br} \gamma$ and [Fe II] luminosities imply a high-mass star formation rate of $0.74 M_{\odot} \mathrm{yr}^{-1}$ or a total star formation rate of $3.9 M_{\odot} \mathrm{yr}^{-1}$ and a SN rate of $0.35 \mathrm{yr}^{-1}$ in the nuclei (Kotilainen et al. 1996), which is similar to the total star formation rate of $3 M_{\odot} \mathrm{yr}^{-1}$ for the Milky Way (Telesco 1988). The radio continuum is predominantly non-thermal betwen $408 \mathrm{MHz}$ and $5000 \mathrm{MHz}$ with a spectral index of -0.77 (PKSCAT90). The system displays the normal radio-FIR correlation which is characteristic of normal and star-forming galaxies. VLA observations at $6 \mathrm{~cm}$ with $4^{\prime \prime}$ resolution by Smith \& Kassim (1993) show emission over 30" and arms of diffuse emission extending out towards the giant tidal arms seen in H I (English et al. 2003). At higher resolution ( $\left.2^{\prime \prime}\right)$, Norris \& Forbes (1995) resolve the nucleus into two equal components, which they argue are the two nuclei of the progenitor galaxies, both undergoing starbursts. From the synchrotron luminosity of the nuclei, they derive a supernova rate of $0.3 \mathrm{yr}^{-1}$ in each nucleus, consistent with estimates from $\mathrm{Br} \gamma$ and [Fe II]. X-ray emission from both nuclei (Lira et al. 2002) is consistent with that from low-luminosity active galactic nuclei (Neff et al. 2003). In summary, the system is a pair of gas-rich disk galaxies that are colliding and hosting a spectacular burst of star formation and AGN activity.

We adopt $H_{0}=75 \mathrm{~km} \mathrm{~s}^{-1} \mathrm{Mpc}^{-1}, q_{0}=0.5$ and $\Lambda=0$, and give velocities in the heliocentric frame using the optical velocity definition throughout this paper.

\section{Observations}

Australia Telescope Compact Array (ATCA) (Frater \& Brooks 1992) observations were made during 1994 Oct. 22 to 24 and 1995 Aug. 05. The ATCA was configured with five antennas on an E-W track with baselines between $46 \mathrm{~m}$ and $750 \mathrm{~m}$.

We observed simultaneously the lines H91 $\alpha$ and H92 $\alpha$ with rest frequencies of $8584.82 \mathrm{MHz}$ and $8309.38 \mathrm{MHz}$, and recorded two orthogonal linear polarizations. We used 64 spectral channels across a $64 \mathrm{MHz}$ bandwidth covering each transition, which corresponds to a velocity coverage of $2270 \mathrm{~km} \mathrm{~s}^{-1}$ with $35 \mathrm{~km} \mathrm{~s}^{-1}$ channel $^{-1}$. We integrated for $24.2 \mathrm{~h}$ on-source.

Calibration and imaging were done using the AIPS software, using standard methods. The flux-density scale assumed that PKS B1934-638 has a flux density of $2.81 \mathrm{Jy}$ at $8236 \mathrm{MHz}$ and 2.66 Jy at $8474 \mathrm{MHz}$, based on the Baars et al. (1977) fluxdensity scale. A phase calibrator was observed every half hour to correct the instrumental phase response. A bandpass calibrator was observed every few hours for correcting the instrumental frequency response (bandpass). Phase corrections obtained from self calibration of the continuum source were applied to the spectral line data. Continuum emission was subtracted from the line data using the method UVLSF (Cornwell et al. 1992), in which the continuum is determined for each baseline by a linear fit to the spectrum. The final continuum and line images were made using robust weighting (robustness 1) of the $(u, v)$ data to achieve near-maximum possible signal-to-noise ratio with resolution of $16.4^{\prime \prime} \times 9.6^{\prime \prime}$ in PA $-14^{\circ}$, which was $20 \%$ higher than with natural weighting. The rms noise level in the spectrum was $130 \mu \mathrm{Jy}_{\text {beam }}^{-1}$ per $1.0 \mathrm{MHz}$ channel after averaging together the two transitions and two polarizations.

Uncertainties on the flux densities have an $11 \%$ rms random multiplicative component due to flux-density bootstrapping and atmospheric opacity, a $0.13 \mathrm{mJy}$ rms random additive component due to thermal noise in a $1 \mathrm{MHz}$ channel or $0.16 \mathrm{mJy}$ rms in the continuum image, and a systematic multiplicative component of $11 \%$ rms due mainly to the uncertainty in the Baars et al. flux-density scale.

Very Large Array (VLA) observations were made in the $\mathrm{CnB}$ configuration on 1998 Nov. 05 and 06 for $8.4 \mathrm{~h}$ with a beamsize of $3.9^{\prime \prime} \times 2.1^{\prime \prime}$ in PA $5^{\circ}$ and in C-array on 1998 Dec. 07 and 1999 Jan. 15 for 3.8 h total, with a beamsize of $12.0^{\prime \prime} \times 2.9^{\prime \prime}$ in PA $10^{\circ}$ (natural weight), to confirm the ATCA observation.

We observed H92 $\alpha$ and recorded two orthogonal circular polarizations. We used 15 spectral channels across a $25 \mathrm{MHz}$ bandwidth in 1998 Nov., which corresponds to a velocity coverage of $910 \mathrm{~km} \mathrm{~s}^{-1}$ with $61 \mathrm{~km} \mathrm{~s}^{-1}$ channel $^{-1}$. We used 15 spectral channels across a $50 \mathrm{MHz}$ bandwidth in $1998 \mathrm{Dec}$. and 1999 Jan., providing twice the velocity coverage.

Data reduction followed the same procedure as for the ATCA data except as follows. The flux-density scale assumed that 3C 286 had a flux density of $5.23 \mathrm{Jy}$ at $8235 \mathrm{MHz}$ (Baars et al. 1977), the phase calibrator served also as bandpass calibrator, and we used natural weighting for the line detection and uniform weighting for best resolution in the continuum image. The rms noise level in the 1998 Nov. spectrum was $81 \mu \mathrm{Jy}$ beam $^{-1}$ per $1.562 \mathrm{MHz}$ channel, and for the combined 1998 Dec. and 1999 Jan. spectrum was $140 \mu \mathrm{Jy} \mathrm{beam}^{-1}$ per $3.125 \mathrm{MHz}$ channel after averaging together the two polarizations. Since the sensitivity of the 1998 Nov. observation was better than the later two observations, and the data could not easily be combined due to their different spectral resolution; the results presented here are based on the 1998 Dec. + 1999 Jan. data since the 1998 Nov. data did not detect line emission.

The observational parameters are summarized in Table 1.

The continuum spectrum was required for separating the thermal and non-thermal continuum components during modelling, and so we used archival VLA B-array continuum observations at $1.4 \mathrm{GHz}$ and $4.8 \mathrm{GHz}$ to complement the ATCA $8.4 \mathrm{GHz}$ measurement. The VLA observations were made on 1990 Oct. 05 in project AS412. The flux-density scale was calibrated assuming $3 \mathrm{C} 48$ had a flux density of $15.62 \mathrm{Jy}$ at $1.4 \mathrm{GHz}$ and $5.49 \mathrm{Jy}$ at $4.8 \mathrm{GHz}$. We tapered the $4.8 \mathrm{GHz}$ array to match the beamsize of the the ATCA at $8.4 \mathrm{GHz}\left(19.9^{\prime \prime} \times\right.$ $\left.10.8^{\prime \prime}\right)$. Since the full-resolution array at $1.4 \mathrm{GHz}$ provided a larger beamwidth $\left(27.6^{\prime \prime} \times 11.3^{\prime \prime}\right)$, we estimated a correction factor to account for the beam mismatch by tapering the $4.8 \mathrm{GHz}$ data to the beamsize of the $1.4 \mathrm{GHz}$ image and calculating the ratio of the peak flux densities measured from the $4.8 \mathrm{GHz}$ images at the two resolutions. This factor, 1.16, was used to scale down the measured $1.4 \mathrm{GHz}$ peak flux density to give an expected value for the smaller beamsize. Since the line 
Table 1. Observational parameters for NGC 3256.

\begin{tabular}{|c|c|c|c|}
\hline Telescope & ATCA & VLA & VLA \\
\hline Date of observing & 1994 Oct. 24 and 1995 Aug. 05 & 1998 Nov. $05 / 06$ & 1998 Dec. $07+1999$ Jan. 15 \\
\hline Array configuration & 750C (1994 Oct.) 375 (1995 Aug.) & $\mathrm{CnB}$ & $\mathrm{C}$ \\
\hline No. antennas & 6 & 27 & 27 \\
\hline Transitions & $\mathrm{H} 92 \alpha \& \mathrm{H} 91 \alpha$ & $\mathrm{H} 92 \alpha$ & $\mathrm{H} 92 \alpha$ \\
\hline Freq. at band centre & $8236 \mathrm{MHz}$ and $8474 \mathrm{MHz}$ & $8233.55 \mathrm{MHz}$ & $8235.1 \mathrm{MHz}$ \\
\hline Beam size & $16.4^{\prime \prime} \times 9.6^{\prime \prime}$ at $-14^{\circ}$ & $\begin{array}{l}3.9^{\prime \prime} \times 2.1^{\prime \prime} \text { in PA } 5^{\circ} a \\
5.7^{\prime \prime} \times 2.6^{\prime \prime} \text { in PA } 1^{\circ} b\end{array}$ & $12.0^{\prime \prime} \times 2.9^{\prime \prime}$ in $\mathrm{PA} 10^{\circ}$ \\
\hline Spectral channels & 64 & 15 & 15 \\
\hline Total bandwidth & $64 \mathrm{MHz}=2270 \mathrm{~km} \mathrm{~s}^{-1}$ & $25 \mathrm{MHz}=910 \mathrm{~km} \mathrm{~s}^{-1}$ & $50 \mathrm{MHz}=1820 \mathrm{~km} \mathrm{~s}^{-1}$ \\
\hline Spectral resolution & $1 \mathrm{MHz}=35 \mathrm{~km} \mathrm{~s}^{-1}$ & $1.562 \mathrm{MHz}=56 \mathrm{~km} \mathrm{~s}^{-1}$ & $3.125 \mathrm{MHz}=112 \mathrm{~km} \mathrm{~s}^{-1}$ \\
\hline$V_{\text {helio,optical }}$ & $2775 \mathrm{~km} \mathrm{~s}^{-1}$ & $2759 \mathrm{~km} \mathrm{~s}^{-1}$ & $2683 \mathrm{~km} \mathrm{~s}^{-1}$ \\
\hline Integration time & $24.2 \mathrm{~h}$ & $8.4 \mathrm{~h}$ & $3.8 \mathrm{~h}$ \\
\hline$T_{\text {sys }}$ & $44 \mathrm{~K}$ & $35 \mathrm{~K}$ (nominal) & $35 \mathrm{~K}$ (nominal) \\
\hline Polarization & dual linear & dual circular & dual circular \\
\hline Phase calibrator & $1104-445$ & $1104-445$ & $1107-448$ \\
\hline Bandpass calibrators & $1104-445,1921-293,2251+158$ & $1104-445$ & $1104-445$ \\
\hline Flux density cal & $1934-638$ & $3 \mathrm{C} 286$ & $3 \mathrm{C} 286$ \\
\hline Assumed flux density & $\begin{array}{l}2.81 \mathrm{Jy} \text { at } 8236 \mathrm{MHz} \\
2.66 \mathrm{Jy} \text { at } 8474 \mathrm{MHz}\end{array}$ & $5.23 \mathrm{Jy}$ at $8235 \mathrm{MHz}$ & $5.23 \mathrm{Jy}$ at $8235 \mathrm{MHz}$ \\
\hline Noise per image channel & $0.13 \mathrm{mJy}$ beam $^{-1}$ & $0.081 \mathrm{mJy}$ beam $^{-1}$ & $0.14 \mathrm{mJy}$ beam $^{-1}$ \\
\hline
\end{tabular}

${ }^{a}$ Continuum image made with uniform weight.

${ }^{b}$ Line image made with natural weight.

emission was unresolved in the ATCA observation, we used the brightness in the continuum images at the position of the peak of the line emission for the continuum flux density for estimating the continuum spectral index. The results are given in Table 2.

\section{Results}

The ATCA continuum and line images, integrated spectrum, and position-velocity diagrams are shown in Figs. 1 to 3. The measured line and continuum parameters are given in Table 2.

The continuum image shows a single, slightly resolved component. The double nuclei detected by Norris \& Forbes (1995) are 4.9" apart and so were not separated by the relatively large ATCA beam. The continuum emission is predominantly non-thermal, with a spectral index of -0.88 between 1.5 GHz and $8.3 \mathrm{GHz}$ (Table 2).

Line emission was detected in the nuclear region, nearly coincident with the peak continuum emission. The line emission region was only slightly resolved with the $16.4^{\prime \prime} \times 9.6^{\prime \prime}$ beam, which implies a size of $\leq 2.9 \mathrm{kpc}$. The offset between the line and continuum peak in Fig. 1 is perhaps significant as it is seen also in the VLA observations. The low-level line emission to the north outside the continuum contours is probably not significant and was also not confirmed by the VLA observations.

The H91 $\alpha+\mathrm{H} 92 \alpha$ spectrum integrated over the compact line-emitting region near the continuum maximum shows a clear line detection at $7.3 \sigma$ with centroid at $2772 \mathrm{~km} \mathrm{~s}^{-1}$, compared to the systemic velocity of $2781 \mathrm{~km} \mathrm{~s}^{-1}$. The line $F W H M$ is $160 \mathrm{~km} \mathrm{~s}^{-1}$.

The position-velocity diagram (Fig. 3) shows the strongest emission near the continuum peak and close to systemic velocity with some complex dynamics in the declination cuts where they intersect the SE extension. The figure shows emission with marginal significance $(3.3 \sigma)$ detected in a southern extension at Dec $=-43^{\circ} 54^{\prime} 28^{\prime \prime}$.

The VLA continuum image and line spectrum are shown in Figs. 4 and 5, and the measured line and continuum parameters are given in Table 2 .

The VLA continuum image with line emission overlayed from 98 Dec. + 99 Jan. is shown in Fig. 4. It shows a $4.0 \sigma$ peak of line emission within the region of continuum emission.

The VLA continuum image at high resolution on 98 Nov. in Fig. 5 has four times higher resolution than the ATCA image and resolves the continuum emission into two nuclei of nearly equal strength separated by $4.8^{\prime \prime}$ ( $870 \mathrm{pc}$ ), consistent with the ATCA continuum image by Norris \& Forbes (1995). The nuclei are resolved, with FWHM diameters of $1.8^{\prime \prime}(320 \mathrm{pc})$ and $2.5^{\prime \prime}$ (450 pc). These also are detected in $\mathrm{H}_{2}, \mathrm{Br} \gamma$, and [Fe II] (Moorwood \& Oliva 1994). Surrounding the two nuclei is a broad low-brightness " $\mathrm{S}$ "-shaped region of radio emission that may be the base of the tidal arms (Kotilainen et al. 1996).

The H92 $\alpha$ line emission (Fig. 6) was detected at $0.56 \mathrm{mJy}$ beam $^{-1}(4.0 \sigma)$ in the $98 \mathrm{Dec} .+99 \mathrm{Jan}$. observation, which confirms the ATCA detection. The peak line strength detected by the VLA appears to be less than that detected by the ATCA. Tapering the VLA data to match the larger beamsize 


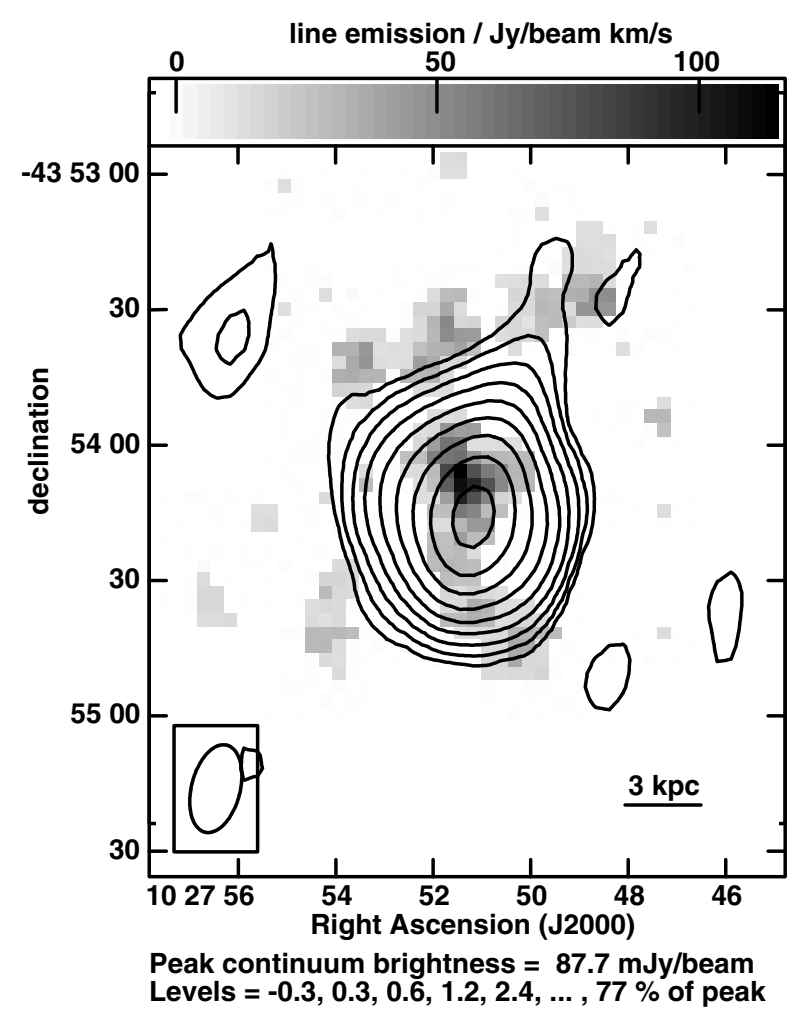

Fig. 1. ATCA $8.4 \mathrm{GHz}$ continuum image of NGC 3256 observed 1994 Oct. +1995 Aug. (contours), superimposed on the grey scale moment 0 image showing $\mathrm{H} 91 \alpha+\mathrm{H} 92 \alpha$ line emission. Beamsize is $16.4^{\prime \prime} \times 9.6^{\prime \prime}$, rms noise is $0.14 \mathrm{mJy}^{\text {beam }}{ }^{-1}$.

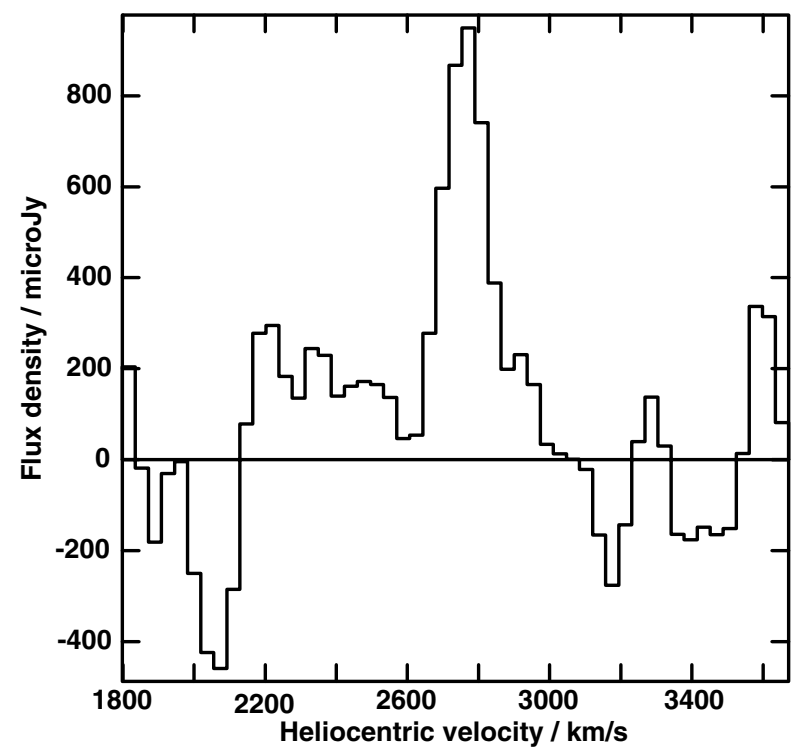

Fig. 2. ATCA H91 $\alpha+\mathrm{H} 92 \alpha$ line profile integrated over the lineemitting region in NGC 3256, observed 1994 Oct. + 1995 Aug. Region of integration is a box of size $24^{\prime \prime} \times 27^{\prime \prime}$ centred on RA 1027 51.303 Dec -43 54 05.7.

of the ATCA yielded a peak line strength of $0.49 \mathrm{mJy}^{\text {beam }}{ }^{-1}$ and so did not reduce the apparent inconsistency. However, the difference between the VLA and ATCA peak line strengths is only $0.9 \sigma$ and so the measurements are formally consistent with each other. The line was not detected in the 98 Nov.

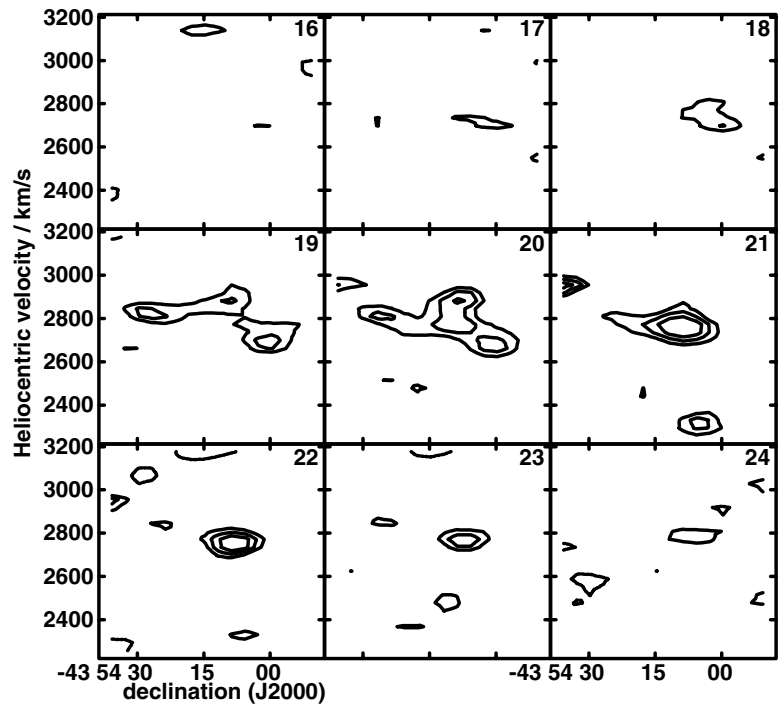

Fig. 3. ATCA position-velocity diagram in NGC 3256 along declination cuts at RA = 102750.33 to 102752.55 (J2000) observed 1994 Oct. +1995 Aug. Contour levels are $-0.5,-0.4,-0.3,0.3,0.4$, $0.5 \mathrm{mJy}$ beam $^{-1}$. The peak flux density is $0.66 \mathrm{mJy}^{\text {beam }}{ }^{-1}$ and the rms noise is 0.13 mJy beam $^{-1}$.

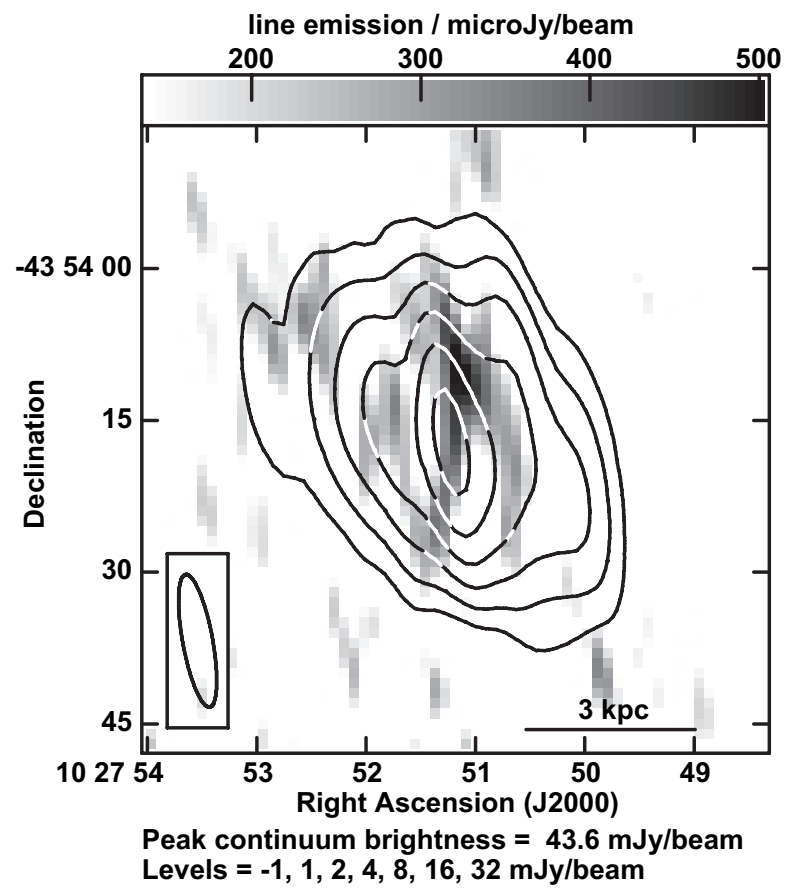

Fig. 4. VLA $8.4 \mathrm{GHz}$ continuum image with natural weight of NGC 3256 observed 1998 Dec. + 1999 Jan. (contours), superimposed on the grey scale image of the channel at $2817 \mathrm{~km} \mathrm{~s}^{-1}$ showing H92 $\alpha$ line emission. Beamsize is $12.0^{\prime \prime} \times 2.9^{\prime \prime}$, rms noise is $0.14 \mathrm{mJy}$ beam $^{-1}$. The grey scale is truncated to white below $1 \sigma$.

observation at either full resolution with natural weight or tapered to match the ATCA beam and we can offer no explanation for this. The $3 \sigma$ upper limit of $0.24 \mathrm{mJy}$ beam $^{-1}$ is lower than the line detections made with the ATCA and with the VLA in the $98 \mathrm{Dec}$. + 99 Jan. observation, but the differences have formal significance of only $1.0 \sigma$ and $2.5 \sigma$. The three observations are therefore formally consistent with each other. 
Table 2. Observational results for NGC 3256.

\begin{tabular}{|c|c|c|c|c|}
\hline Parameter & $\mathrm{ATCA}^{a}$ & VLA (north nucleus) & VLA (south nucleus) & VLA (98 Dec. + 99 Jan.) \\
\hline \multicolumn{5}{|l|}{ Continuum properties } \\
\hline Peak position RA (J2000) & 102751.182 & 102751.2199 & 102751.1946 & 102751.2296 \\
\hline Peak position declination & -435415.89 & -435413.79 & -435418.61 & -435417.26 \\
\hline Peak flux density $\left(\mathrm{mJy}\right.$ beam $\left.{ }^{-1}\right)$ & $89.1 \pm 13$ & $15.9 \pm 2.5$ & $15.1 \pm 2.4$ & $43.4 \pm 6.8$ \\
\hline Integrated flux density (mJy) & $152 \pm 24$ & $25.4 \pm 4.0$ & $27.8 \pm 4.3$ & $156 \pm 24$ \\
\hline Deconvolved size & $13.3^{\prime \prime} \times 10.6^{\prime \prime}$ at $47^{\circ}$ & $2.3^{\prime \prime} \times 1.4^{\prime \prime}$ at $8^{\circ}$ & $2.8^{\prime \prime} \times 2.2^{\prime \prime}$ at $42^{\circ}$ & $5.0^{\prime \prime} \times 3.8^{\prime \prime}$ at $10^{\circ}$ \\
\hline \multicolumn{5}{|l|}{ Line properties } \\
\hline Peak line flux density (mJy beam ${ }^{-1}$ ) & $0.95 \pm 0.27$ & $<0.24$ & $<0.24$ & $0.56 \pm 0.32$ \\
\hline Velocity dispersion $\left(\mathrm{km} \mathrm{s}^{-1}\right)$ & $87 \pm 26$ & - & - & $48 \pm 25$ \\
\hline Integrated line flux $\left(\mathrm{Jy} \mathrm{km} \mathrm{s}^{-1}\right)$ & $0.16 \pm 0.05$ & - & - & $0.05 \pm 0.03$ \\
\hline Integrated line flux $\left(10^{-23} \mathrm{~W} \mathrm{~m}^{-2}\right)$ & $4.3 \pm 1.3$ & - & - & $1.4 \pm 0.8$ \\
\hline Cont. flux density over line region (mJy) & $116 \pm 18$ & - & - & $19 \pm 3$ \\
\hline Centroid helio. optical vel. $\left(\mathrm{km} \mathrm{s}^{-1}\right)$ & $2772 \pm 22$ & - & - & $2817 \pm 36$ \\
\hline No. of beam areas where line is observed & 0.1 & - & - & 1.0 \\
\hline Line width $(F W H M)$ in $\left.\mathrm{km} \mathrm{s}^{-1}\right)$ & $161 \pm 48$ & - & - & $114 \pm 60$ \\
\hline Distance & $37 \mathrm{Mpc}$ & & & \\
\hline \multicolumn{5}{|l|}{ Other measurements } \\
\hline Continuum flux density at: & & & & \\
\hline $1.49 \mathrm{GHz}\left(\mathrm{mJy} \mathrm{beam}^{-1}\right)$ & $211^{b}$ & & & \\
\hline $4.90 \mathrm{GHz}\left(\mathrm{mJy}\right.$ beam $\left.^{-1}\right)$ & 119 & & & \\
\hline 8.30 GHz (mJy beam $\left.{ }^{-1}\right)$ & 49 & & & \\
\hline Estimated thermal em. at $8.3 \mathrm{GHz}(\mathrm{mJy})$ & 26 & & & \\
\hline $\operatorname{Br} \gamma$ flux $\left(\mathrm{W} \mathrm{m}^{-2}\right)$ & $5.3 \times 10^{-17 c}$ & & & \\
\hline
\end{tabular}

${ }^{a}$ These ATCA measurements were used in the radiative transfer modelling.

${ }^{b}$ Beamwidth $=19.9^{\prime \prime} \times 10.8^{\prime \prime}$.

${ }^{c} 6^{\prime \prime}$ aperture, Kotilainen et al. (1996).

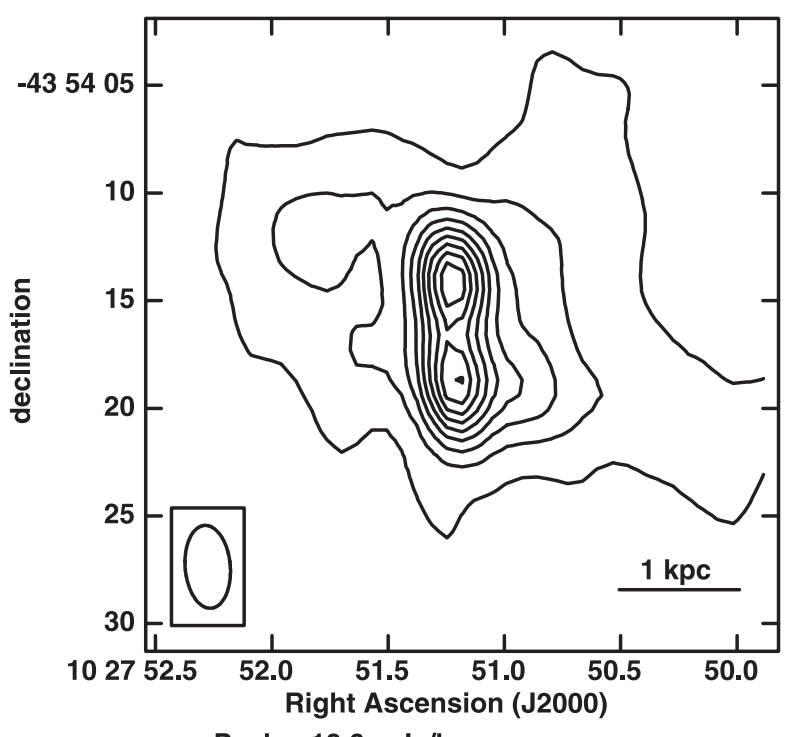

Peak = $18.6 \mathrm{mJy} / \mathrm{beam}$

Contours at $-1,1,3,5, \ldots, 17 \mathrm{mJy} / \mathrm{beam}$

Fig. 5. VLA $8.4 \mathrm{GHz}$ continuum image at higher resolution, with uniform weight of NGC 3256 observed 1998 Nov., showing the double nucleus. Beamsize is $3.9^{\prime \prime} \times 2.1^{\prime \prime}$, rms noise is $0.10 \mathrm{mJy} \mathrm{beam}^{-1}$.

Emission is centred near $2800 \mathrm{~km} \mathrm{~s}^{-1}$, near the systemic velocity. This narrow RRL component is coincident in velocity

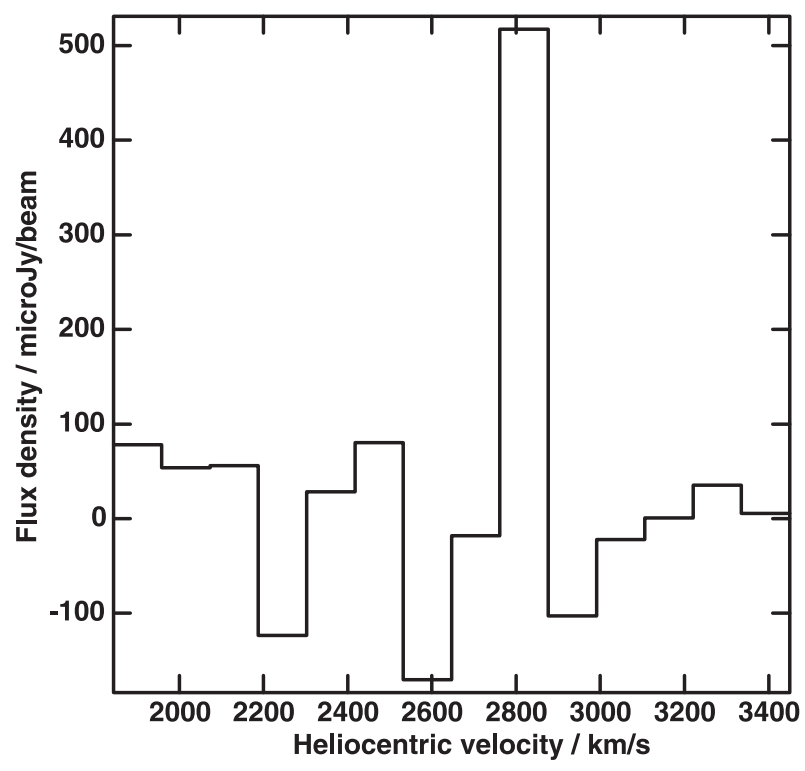

Fig. 6. VLA H92 $\alpha$ line profile at the peak of the line emission in the nucleus NGC 3256, observed 1998 Dec. + 1999 Jan., observed with a $50 \mathrm{MHz}$ bandwidth. Beamwidth is $12.0^{\prime \prime} \times 2.9^{\prime \prime}$ in PA $4^{\circ}$ and does not resolve the double nuclei.

with the $\mathrm{H} \mathrm{I}$ absorption towards the nucleus seen by English et al. (2003), which spans $2700 \mathrm{~km} \mathrm{~s}^{-1}$ to $2960 \mathrm{~km} \mathrm{~s}^{-1}$. 
Table 3. Properties of the ionized gas in both nuclei in NGC 3256 derived from the ATCA line detection using the Collection of H II regions model.

\begin{tabular}{ll}
\hline \hline Parameter & Value \\
\hline Source & NGC 3256 \\
Electron temperature & $5000 \mathrm{~K}$ \\
Electron density & $1000 \mathrm{~cm}^{-3}$ to $5000 \mathrm{~cm}^{-3}$ \\
Number of HII regions & 10 to 300 \\
Size & $15 \mathrm{pc}$ \\
Total ionized gas mass & $4 \times 10^{4} M_{\odot}$ to $2 \times 10^{5} M_{\odot}$ \\
$N_{\text {LyC }}$ & $2 \times 10^{52} \mathrm{~s}^{-1}$ to $6 \times 10^{53} \mathrm{~s}^{-1}$ \\
No. O5 stars & 600 to 17000 \\
Fraction of thermal continuum at $5 \mathrm{GHz}$ & $1 \%$ to $7 \%$ \\
Filling factor & $3 \times 10^{-4}$ to $8 \times 10^{-3}$ \\
\hline
\end{tabular}

\section{Modelling the ionized gas}

Conditions in the ionized gas are constrained by the observed line and continuum emission. Following Anantharamaiah et al. (1993), we consider two simple models: 1) a uniform slab of ionized gas in front of the central non-thermal continuum source; and 2) a collection of H II regions within the central few-hundred parsecs.

1. The slab model consists of a slab of ionized gas with the same lateral size as the line-emitting region, and is characterized by an electron temperature $T_{\mathrm{e}}$, electron density $n_{\mathrm{e}}$ and thickness along the line of sight $l$. The peak line flux density is then given by Eq. (1) of Anantharamaiah et al. (1993) and contains contributions from spontaneous emission from the slab, stimulated emission amplifying thermal emission within the slab, and stimulated emission amplifying the background continuum. Thermal continuum emission from the slab is readily derived given $T_{\mathrm{e}}, n_{\mathrm{e}}$ and $l$. We found that there are no uniform-slab models that fit the observed line and continuum flux densities simultaneously and so we do not consider this model further.

2. The collection of H II regions model consists of a collection of spherical H II regions, all with the same $T_{\mathrm{e}}, n_{\mathrm{e}}$ and linear diameter $l$ embedded in a volume of uniform synchrotron emission. The total number of clouds, $N$, is determined by calculating the line flux density produced by a single cloud with the given conditions and dividing that into the total observed line strength. Some combinations of conditions can be ruled out using the following requirements. 1) The volume filling factor of H II regions, calculated by dividing the volume of the line-emitting region by $N$ times the volume of an individual $\mathrm{H}$ II region, should not exceed unity. 2) Since the line width of a single H II region is much less than the observed line width, a minimum number of $\mathrm{H}$ II regions with different velocities must exist inside every beam are within the line-emitting region. 3) The peak line flux density of a single H II region should not exceed the observed line flux density. 4) The total thermal continuum emission from the H II regions, $S_{\text {th }}$, should not exceed that inferred from the $\mathrm{Br} \gamma$ flux.
We considered a grid of models with $T_{\mathrm{e}}, n_{\mathrm{e}}$ and $l$ in the ranges $1000 \mathrm{~K}$ to $12500 \mathrm{~K}, 10 \mathrm{~cm}^{-3}$ to $10^{6} \mathrm{~cm}^{-3}$ and $0.01 \mathrm{pc}$ to $100 \mathrm{pc}$. Models with 10 to $300 \mathrm{H}$ II regions, all with $T_{\mathrm{e}} \sim 5000 \mathrm{~K}$, $n_{\mathrm{e}} \sim 10^{3} \mathrm{~cm}^{-3}$ to $10^{4} \mathrm{~cm}^{-3}$ and size $l \sim 15 \mathrm{pc}$ produced good matches to the line and continuum emission.

Parameters derived for typical allowed models are given in Table 3.

\section{Discussion}

The strength of the RRL emission infers a mass of ionized gas of $4 \times 10^{4} M_{\odot}$ to $2 \times 10^{5} M_{\odot}$, depending on the model conditions, which requires a Lyman continuum flux of $2 \times 10^{52} \mathrm{~s}^{-1}$ to $6 \times 10^{53} \mathrm{~s}^{-1}$ to maintain the ionization. This flux is equivalent to the Lyman continuum output of 600 to 17000 stars of type 05 .

This ionized gas, if spread over two nuclei of $320 \mathrm{pc}$ and $450 \mathrm{pc}$ diameter has a surface density of $(0.2$ to 0.8$) M_{\odot} \mathrm{pc}^{-2}$, compared to the star formation threshold found by Kennicutt (1989) of (3 to 10) $M_{\odot} \mathrm{pc}^{-2}$ for the total gas mass.

The molecular gas mass inferred from $\mathrm{CO}$ emission by Sargent et al. (1989), is $10^{5}$ times larger than the ionized gas mass we derive from the RRLs, and if the molecular gas is spread over the central kiloparsec, the surface density of $40000 M_{\odot} \mathrm{pc}^{-2}$ far exceeds Kennicutt's star formation threshold. Thus, of the vast quantity of molecular gas present, only a tiny fraction has been ionized in the starburst.

The expected X-ray luminosity of 17000 stars of type O5, adopting $10^{26.3} \mathrm{~W}$ per O5 star (Chlebowski et al. 1989) is $3.4 \times$ $10^{30} \mathrm{~W}$, which is a factor 1000 less than the observed X-ray luminosity from the two compact X-ray components that are coincident with the radio components, namely ULX $7(\mathrm{~N})$ and ULX 8(S). Thus, we confirm the conclusion of Neff et al. (2003) that the star formation activity is insufficient to account for the nuclear X-ray emission.

The observed dereddened $\mathrm{Br} \gamma$ flux is $1.54 \times 10^{-16} \mathrm{~W} \mathrm{~m}^{-2}$ (Prestwich et al. 1994) which agrees with that predicted from the measured RRL strength, $(0.3$ to 1.5$) \times 10^{-16} \mathrm{~W} \mathrm{~m}^{-2}$, depending on the model parameters. The dereddened $\operatorname{Br} \alpha$ flux is $13.5 \times 10^{-16} \mathrm{~W} \mathrm{~m}^{-2}$ (Rigopoulou et al. 1996), yielding a ratio 
of $\operatorname{Br} \alpha$ to $\operatorname{Br} \gamma$ of 8.8 , which is different from the expected 2.96 assuming case $\mathrm{B}$ recombination. This discrepancy could be due to the use of different aperture sizes and extinction models for measuring and dereddening the different transitions, or due to the simple foreground screen model not being accurate in the case of clumpy heavy extinction.

We infer an electron density of $5000 \mathrm{~cm}^{-3}$ within the $\mathrm{H}$ II regions in the central starburst, though values in the range $1000 \mathrm{~cm}^{-3}$ to $10000 \mathrm{~cm}^{-3}$ are also allowed by the available constraints from the RRL detection. This high density at first appears to be in conflict with the electron density of $300 \mathrm{~cm}^{-3}$ derived by Rigopoulou et al. (1996) derived from the [S III] line ratios, and with the limit of $<1000 \mathrm{~cm}^{-3}$ established by Carral et al. (1994) using the [O III] line ratios. However, Anantharamaiah et al. (2000) point out that lower values from the [S III] method may be a selection effect caused by the insensitivity of the $[\mathrm{S} \mathrm{III]} \mathrm{ratios} \mathrm{to} \mathrm{densities} \mathrm{outside} \mathrm{the} \mathrm{range}$ $\left(10^{2}\right.$ to $\left.10^{3.5}\right) \mathrm{cm}^{-3}$ (Houck et al. 1984).

\section{Conclusions}

We have detected $\mathrm{H} 91 \alpha$ and $\mathrm{H} 92 \alpha$ lines in emission in NGC 3256 using the ATCA and the VLA with a width of $160 \mathrm{~km} \mathrm{~s}^{-1}$ (measured with the ATCA) and $114 \mathrm{~km} \mathrm{~s}^{-1}$ (measured with the VLA). The line emission region is coincident with the continuum emission originating in the nuclei and its integrated flux infers a mass of ionized gas of $4 \times 10^{4} M_{\odot}$ to $2 \times 10^{5} M_{\odot}$, requiring 600 to 1700005 stars to maintain the ionization.

Acknowledgements. The National Radio Astronomy Observatory is a facility of the National Science Foundation operated under cooperative agreement by Associated Universities, Inc. The Australia Telescope Compact Array is part of the Australia Telescope, which is funded by the Commonwealth of Australia for operation as a National Facility managed by CSIRO.

\section{References}

Anantharamaiah, K. R., \& Goss, W. M. 1997, RMxAC, 6, 58 Anantharamaiah, K. R., Zhao, J. H., Goss, W. M., \& Viallefond, F. 1993, ApJ, 419, 585
Anantharamaiah, K. R., Viallefond, F., Mohan, N. R., Goss, W. M., \& Zhao, J. H. 2000, ApJ, 537, 613

Bell, M. B., \& Seaquist, E. R. 1978, ApJ, 223, 378

Bell, M. B., Seaquist, E. R., Mebold, U., Reif, K., \& Shaver, P. A. 1984, A\&A, 130, 1

Carral, P., Hollenbach, D. J., Lord, S. D., et al. 1994, ApJ, 423, 223

Chlebowski, T., Harnden, F. R., \& Sciortino, S. 1989, ApJ, 341, 427

Cornwell, T. J., Uson, J. M., \& Haddad, N. 1992, A\&A, 258, 583

de Vaucouleurs, et al. 1991, Third Reference Catalogue of Bright Galaxies, version 3.9

English, J., Norris, R. P., Freeman, K. C., \& Booth, R. C. 2003, AJ, 125,1134

Forbes, D. A., Norris, R. P., Williger, G. M., \& Smith, R. C. 1994, AJ, 107, 984

Frater \& Brooks 1992

Glass, I. S., \& Moorwood, A. F. M. 1985, MNRAS, 214, 429

Helou, G., Soifer, B. T., \& Rowan-Robinson, M. 1985, ApJ, 298, L7

Houck, J. R., Shure, M. A., Gull, G. E., \& Herter, T. 1984, ApJ, 287, L11

Kennicutt, R. C. J. 1989, ApJ, 344, 685

Kotilainen, J. K., Moorwood, A. F. M., Ward, M. J., \& Forbes, D. A. 1996, A\&A, 305, 107

Kulkarni, S. R., \& Heiles, C. 1988, in Galactic and Extragalactic Radio Astronomy, ed. G. L. Verschuur, \& K. I. Kellermann

Lira, P., Ward, M., Zezas, A., Alonso-Herrero, A., \& Ueno, S. 2002, MNRAS, 330, 259

Mohan, Niruj R., Anantharamaiah, K. R., \& Goss, W. M. 2001, ApJ, 557,659

Moorwood, A. F. M., \& Oliva, E. 1994, ApJ, 429, 602

Neff, S. G., Ulvestad, J. S., \& Campion, S. D. 2003, ApJ, 599, 1043

Norris, R. P., \& Forbes, D. A. 1995, ApJ, 446, 594

Phookun, B., Anantharamaiah, K. R., \& Goss, W. M. 1998, MNRAS, 295, 156

Prestwich, A. H., Joseph, R. D., \& Wright, G. S. 1994, ApJ, 422, 73

Rigopoulou, D., Lutz, D., Genzel, R., et al. 1996, A\&A, 315, L125

Rowan-Robinson, M., \& Crawford, J. 1989, MNRAS, 238, 523

Sargent, A. I., Sanders, D. B., \& Phillips, T. G. 1989, ApJ, 346, L9

Schweizer, F. 1986, Science, 231, 227

Seaquist, E. R., \& Bell, M. B. 1977, A\&A, 60, L1

Shaver, P. A. 1978, A\&A, 68, 97

Shaver, P. A., Churchwell, E., \& Rots, A. H. 1977, A\&A, 55, 435

Telesco, C. M. 1988, ARA\&A, 26, 343

Zhao, J.-H., Anantharamaiah, K. R., Goss, W. M., \& Viallefond, F. 1996, ApJ, 472, 54 